\title{
Actualizando los abordajes socioculturales de la obesidad: propuestas a partir de Hacking, Bourdieu y Foucault
}

\author{
I 1 José Pedro Hernández Alcaraz, ${ }^{2}$ José Palacios Ramírez, \\ ${ }^{3}$ María Esther López Peinado I
}

Resumen: El aumento de las tasas de obesidad y sus consecuencias negativas, tanto individuales como colectivas, reclama la necesidad de nuevos enfoques científicos, que permitan un abordaje multidisciplinar que integre los factores biológicos y socioculturales, explorando en profundidad tanto los aspectos objetivos como subjetivos de este problema. En este sentido, en el presente trabajo proponemos un conjunto de reflexiones teóricas, que puedan servir como marco crítico de actualización de las aportaciones de corte sociocultural en los debates científicos sobre la obesidad y el sobrepeso. Para ello, primero revisaremos las principales características de los enfoques biológico y sociocultural sobre el tema. Y después pasaremos a proponer dicho conjunto de reflexiones, realizando un análisis de los conceptos de tres teóricos sociales de corte crítico, vistos como modelos potencialmente fértiles pero poco usados en este tipo de tema por distintos motivos.

> Palabras clave: obesidad; debates científicos; enfoques socioculturales; Hacking; Bourdieu; Foucault; actualización teórica.

\author{
1 Programa de las Ciencias de \\ la Salud, Universidad Católica \\ de Murcia. Murcia, España \\ (josepe189@gmail.com). \\ ORCID: 0000-0002-6180-215X \\ ${ }^{2}$ Facultad de C. de la Salud, \\ Universidad Católica de Murcia. \\ Murcia, España (jpalacios@ \\ ucam.edu). \\ ORCID: 0000-0001-8607-6425 \\ ${ }^{3}$ Consejería de Educación y \\ cultura, Región de Murcia. \\ Murcia, España (lopezpeinado. \\ mesther@gmail.com). \\ ORCID: 0000-0002-1682-4632
} Aprovado em: 29/05/2020 Revisado em: 03/09/2020 


\section{Introducción: un problema complejo}

Aunque en ocasiones lo parezca, la obesidad no puede considerarse exactamente un problema nuevo. A través de la Historia, el Arte y la Arqueología, sabemos que el exceso de peso ha acompañado al ser humano durante toda su evolución. Considerada en la mayoría de períodos y contextos culturales como un signo de distinción social (BROWN, 1991), es desde finales del s. XX cuando va adquiriendo creciente notoriedad como problema público de salud. Aunque en distintos momentos de la extensa tradición médica occidental pueden encontrarse elementos que sirven de base para las actuales concepciones clínicas del problema, como el Índice de Masa Corporal, el interés por su base genética o por su vínculo con la compulsividad. Las condiciones para su eclosión como problema social vendrán marcadas por los cambios acaecidos fruto del despliegue de un sistema alimentario global, en diferentes etapas que van desde el auto-abastecimiento (MONTANARI, 2006) y las carencias nutricionales generadas por la Segunda Guerra mundial (BENGOA, 2003), hasta los procesos de occidentalización de las prácticas alimenticias debido a la influencia del marketing y el abaratamiento de los alimentos procesados (LANGREO, 2005). Dándose lo que Fishler (1995) denomina como anomia alimentaria, es decir, un alejamiento progresivo de los modelos tradicionales de alimentación y sus marcos ecológico-culturales.

En este complejo contexto social, la Organización Mundial de la Salud (OMS) elabora en el año 2000 un informe titulado "Obesidad: Prevención y Gestión de una Epidemia Mundial”, en el que la obesidad es denominada como epidemia global debido a su presencia en la mayoría de los países a nivel mundial, llegando incluso a convivir en algunos entornos con la desnutrición: "En muchos países en desarrollo que se encuentran en fase de transición económica, a menudo coexisten en la misma población (o incluso en la misma familia) niveles crecientes de obesidad con desnutrición crónica” (OMS, 2003, p. 68).

Estas circunstancias han propiciado nuevos debates y puntos de vista entre los que destaca el enfoque "ambiental", cuya hipótesis es que los nuevos ambientes obesogénicos juegan en contra de la herencia genética humana (ULIJASZEK; LOFINK, 2006). No obstante, los enfoques biomédicos han copado las explicaciones oficiales de la obesidad, manteniendo un discurso que pone el foco sobre los riesgos e imprudencias de aquellos sujetos que se dejan llevar por sus instintos y cuya genética 
les predispone a padecer obesidad en contextos de abundancia de alimentos; mientras que se ensombrecen otras cuestiones como los esquemas de valores, percepciones y creencias culturales que también forman parte de este complejo engranaje biopsico-social (GRACIA, 2009). Paralelamente, observamos el protagonismo que van cobrado las Ciencias sociales y humanísticas en las investigaciones médicas, por su capacidad para identificar campos y problemas emergentes que requieren de la comprensión de determinados aspectos subjetivos de las relaciones personales (FLORES; MARIANO, 2016). Todo esto invita a pensar que nos encontramos en un momento adecuado para plantear un giro epistemológico que realce la importancia de un enfoque sociocultural centrado en las ideas que envuelven a la obesidad y las relaciones interactivas de los sujetos con dichas ideas.

Con nuestra propuesta planteamos una reflexión sobre el aporte de estos conocimientos socioculturales (BERTRAN, 2010) en la comprensión del sobrepeso y/o la obesidad, pues si queremos conseguir intervenciones efectivas en este área entendemos que es necesario desarrollar colaboraciones entre profesionales de diferentes ámbitos (LAKE; TOWNSHEND, 2006), pero también reflexionar sobre la posibilidad de incluir otras formas de entender qué es eso de lo sociocultural, qué relación debe establecerse con los otros componentes de la tríada bio-psico-social o los equilibrios entre crítica y aplicación.

\section{Modelos explicativos predominantes}

Para comprender mejor los planteamientos que proponemos, puede ser de gran utilidad realizar un análisis comparativo y diacrónico de cómo las diferentes corrientes han explicado el exceso de peso hasta el momento, mostrando una síntesis de las ideas fundamentales que defienden los marcos explicativos vigentes: biomédico y sociocultural. Seguidamente, desarrollaremos algunos retos teóricos implícitos que se presentan a los enfoques socioculturales ante una posible integración progresiva entre ambas perspectivas.

\section{Definiciones y abordajes biomédicos}

Podríamos remontarnos a la Antigua Grecia para encontrar la base de lo que se puede considerar como la explicación del exceso de peso de la corriente biomédica. Así, Foucault (1991) detecta las primeras formas de gobierno de la vida pública a 
través de la medicina cuando Hipócrates sugiere que la clave para adelgazar reside en la temperancia, es decir, en el autocontrol de los individuos. Una lectura del fenómeno aún vigente en la actualidad como se puede observar en los discursos de algunos expertos que consideran a las personas obesas responsables de un consumo irracional de alimentos y de llevar un estilo de vida sedentario (ÁLVAREZ, 2010).

En estalínea, la máxima hipocrática: "que tu comida sea tu medicina" (MANZINI, 1996), podría considerarse como el precedente de la "medicalización de la vida”, un fenómeno que se refiere a: "[...] la forma en que el ámbito de la medicina moderna se ha expandido en los años recientes y ahora abarca muchos problemas que antes no estaban considerados como entidades médicas" (MÁRQUEZ; MENEU, 2003, p. 65). La cuestión es que esta estrategia se ha convertido en uno de los pilares de los enfoques biomédicos, especialmente tras importantes acontecimientos históricos que podemos sintetizar en: el surgimiento de la medicina moderna del siglo XVIII, época en la que el cuerpo humano se comienza a ver como una fuerza productiva, en el marco de una economía capitalista (FOUCAULT, 1996) y la explosión del positivismo en el siglo XIX (COMTE; REYES, 1979) que ha cosificado el cuerpo a sus componentes biológicos.

Actualmente podemos considerar también los cambios estructurales acaecidos, como la hiper-producción de alimentos, el desarrollo de la publicidad, el sedentarismo o el hiper-desarrollo de los medios de transporte y las comunicaciones. Circunstancias que han propiciado entornos que algunos autores han denominado "tóxicos" por la forma en que favorecen el exceso de peso y que la perspectiva biomédica enfoca como una inadaptación del patrimonio evolutivo del ser humano a dichos contextos (FRENCH et al., 2001; BROW NELL, 2002). Bajo esta perspectiva Swinburn, Egger y Raza (1999), acuñaron el concepto de ambiente obesogénico para explicar la forma en que las sociedades modernas crean entornos económicos, sociales y culturales propicios para un desequilibrio energético. Este concepto encajaba además con otro elemento con cierto peso específico dentro del enfoque biomédico: la causalidad genética. En este campo, Neel (1962) defendía con su teoría del "gen ahorrador" que la capacidad del ser humano de acumular grasa con gran facilidad, podría haber actuado como un factor de selección natural a favor de la supervivencia de nuestros antepasados, debido a la frecuencia de las épocas de escasez de alimentos. Mientras que actualmente este genotipo resultaría desventajoso, especialmente en ambientes proclives al consumo excesivo de alimentos. 
A pesar de haber transcurrido décadas desde estas primeras ideas sobre la relación entre genética y obesidad, este campo sigue vigente debido a la irrupción del concepto de epigenética. Según este nuevo enfoque la obesidad se podría transmitir de padres a hijos a través de mecanismos que no implican necesariamente cambios en la secuencia del ADN (ácido desoxirribonucleico), sino más bien en los mecanismos mediante los cuales se expresan los genes y que se activarían por medio de "gatillos" ambientales ligados al estilo de vida o a la articulación de la exposición a diferentes factores (CORELLA; COLTELL; ORDOVAS, 2016).

\section{El enfoque sociocultural: un primer desarrollo}

En líneas generales, puede decirse que la perspectiva sociocultural sobre la obesidad se asienta sobre la base de que la alimentación es un "hecho social total". Una noción acuñada por Mauss (1971), que hacía referencia a la forma en que a través de la alimentación se expresan todo tipo de instituciones:

\footnotetext{
[...] las religiosas, jurídicas, morales y económicas, las cuales adoptan formas especiales de producción y consumo [...], y a las cuales hay que añadir los fenómenos estéticos a que estos hechos dan lugar, así como los fenómenos morfológicos que estas instituciones producen (MAUSS, 1971, p. 157).
}

Otros ejemplos de los primeros avances de este enfoque serían las aportaciones de Levi- Strauss (1868), sobre las transformaciones culturales metaforizadas en el paso del consumo de alimentos crudos a alimentos cocidos. La obra de Mennell (2009), donde se relacionan las normas que regulan la forma de alimentarse con grupos sociales determinados. O la de Elias (1989), que estudia la incorporación de normas y modales en la mesa en relación al proceso civilizador que habría tenido lugar entre los siglos XIII y XIX en Occidente, mediante.

En posteriores avances surgen enfoques socioculturales que sugieren explicaciones multicausales, que requieren de un análisis más complejo, e incluyan aspectos como los cambios socio-económicos o las estrategias que las personas adoptan para solucionar sus problemas de salud en base a sus creencias y valores (GRACIA, 2010). Otra cuestión señalada desde los enfoques socioculturales más actuales es que la alarma en torno al problema, esté legitimando estrategias que implican el disciplinamiento de los cuerpos y las conciencias individuales, y a su vez generando cierta dependencia de las instancias expertas (BOERO, 2007). 
En resumen, mientras que las concepciones biomédicas entrecruzan lógicas clínicas y morales descargando en el sujeto obeso la responsabilidad de sobreponerse a los entornos tóxicos para preservar su salud y peso corporal (SAGUY; RILEY, 2005). Las perspectivas del ámbito socio-cultural problematizan los límites entre lo natural y lo cultural, reclamando que los enfoques biomédicos eluden ciertos niveles de complejidad que van más allá de los determinismos ecológicos, biológicos o psicológicos, sin comprender la heterogeneidad de los marcos socioculturales que dificultan el seguimiento de las prescripciones dietéticas (GRACIA, 2007).

\section{Actualizar los aportes de corte sociocultural en torno a la obesidad: reflexiones a partir de Hacking, Bourdieu y Foucault}

La incorporación de los conocimientos socioculturales a las discusiones actuales ha sido limitada, en parte por causas de carácter "paradigmático", como su "inadecuación" teórico-metodológica de los aportes sociales ante un marco constituido sobre presupuestos positivistas y biologicistas. En parte también debido a limitaciones autoimpuestas en base a sus propias lógicas disciplinares. Esta articulación de marcos epistemológicos distintos, parece haber devenido en una complementariedad con cierto sentido de status quo, que parece seguir reservando una cierta hegemonía para las concepciones biológicas. Sin duda nuestra propuesta debe enmarcarse en el reconocimiento de una serie transformaciones, que atraviesan el campo de los conocimientos sociales sobre la salud y que tienen que ver con cambios en las relaciones entre crítica y aplicación en áreas como la antropología o la sociología de la salud, que paulatinamente han pasado de considerarse ámbitos sin capacidad de teorizar, a ámbitos centrales en la producción de teoría en torno a problemáticas y debates públicos de actualidad para las sociedades contemporáneas (NUNES, 2014).

En este sentido, el objetivo de este ensayo es realizar un aporte actualizador sobre el potencial de los modelos socioculturales en las nombradas dimensiones biopsico-sociales de la salud. Para ello nos apoyaremos en tres autores, los filósofos Ian Hacking y Michel Foucault, y el sociólogo Pierre Bourdieu, cuyas ideas presentan a nuestro entender un enorme potencial para su aplicación a la problemática de la obesidad en nuestras sociedades. Pese a ser autores sobradamente conocidos en las Ciencias Sociales de los últimos años, su aplicación en ámbitos concretos de la 
antropología o la sociología de la salud ha sido mucho más limitada, especialmente en cuanto a su operacionalización empírica respecto a problemas específicos como el que nos ocupa. Esperamos que estas páginas sirvan como espacio de contraste de ideas no solo para los familiarizados con ellos, sino sobre todo para los investigadores más afines a posiciones biomédicas que puedan desconocerlos.

$\mathrm{Si}$ bien es cierto que se trata de tres autores con backgrounds disciplinares y focos de interés relativamente alejados, al mismo tiempo existen entre ellos un conjunto de conexiones epistemológicas que pueden permitir establecer articulaciones entre sus conceptos. En cierto modo los tres parten de posiciones postestructuralistas (FERNÁNDEZ DE ROTA, 2008), de un realismo filosófico respecto a las entidades que analizan que les permite reconocer la positividad de determinados conocimientos científicos a la vez que cuestionar sus pretensiones universalistas, preguntándose por sus efectos sociales en términos de autoridad, poder o control social. Otro punto de encuentro entre estos tres autores sería el entendimiento productivo y no restrictivo de las relaciones de poder. Tanto Hacking como Foucault parten de perspectivas filosóficas e históricas sobre los desarrollos de la ciencia occidental (singularmente Medicina, Psiquiatría o Criminología), significativos en cuanto a su problematización/conformación de la naturaleza humana, que exigen de mayor "bricolaje" teórico para su aplicación empírica. Mientras en el caso de Bourdieu, aun existiendo también una base filosófica en torno a las racionalidades prácticas, sus desarrollos empíricos facilitan mucho más su aplicación concreta.

\section{¿Qué nos pueden aportar estos autores?}

Aunque los trabajos de I. Hacking (2001) no han sido aplicados al estudio del exceso de peso, entendemos que su crítica del constructivismo social provee de un marco extraordinario para la reflexión sobre los desórdenes alimentarios, toda vez que parece tan necesario ponderar tanto el profundo biologicismo predominante hasta hoy en el entendimiento del problema, como la tentación constructivista de una crítica fácil que no atienda a la gravedad real de la cuestión. Entender la obesidad como una categoría interactiva, implicaría atender a los efectos que genera en las personas que sostienen dicha categoría, así como en aquellos que asisten y/o participan de dicho etiquetamiento. Desde su interés por los efectos sociales de las prácticas científicas, el trabajo de Hacking resulta interesante para captar lo que denomina formas prácticas de ajuste entre los discursos preventivos, los diagnósticos 
expertos, las prácticas profanas de auto-intervención y el carácter estigmatizador de dichos diagnósticos (HACKING, 2001, p. 60-61).

En el caso de P. Bourdieu, el uso de sus conceptos en investigaciones relativas al exceso de peso y la alimentación es más extenso, pues proveen de un marco empírico capaz de abordar cuestiones como la conformación de los gustos alimentarios en distintos segmentos de clase o los procesos de imitación y apropiación respecto a algunos de dichos segmentos (SATO et al., 2016). Podemos destacar su aplicación en estudios como el de Murrieta (1998), que observa cómo la alimentación dentro de una comunidad amazónica está marcada por la voluntad de los individuos de distinguirse. Y también el trabajo de Martín y Moreno (2005) en el que describen el modo en que las diferentes concepciones de lo sano atraviesan el campo social de la alimentación, destacando la importancia de las estructuras familiares o el papel estratégico de los capitales culinarios.

Bajo el prisma de M. Foucault, la obesidad emerge como un problema definido en un marco socio-históricamente dado de relaciones de conocimiento y poder. Tomando como referencia la reorientación de sus teorías posterior al conocido Vigilar y castigar, el foco se situaría en el despliegue de marcos colectivos e individuales de regulación (estéticos, morales, políticos, saludables) sobre las conductas alimentarias y/o los deseos y prácticas corporales, que se moverían entre las determinaciones externas y las formas de auto-perfeccionamiento constituidas en relación a los modelos dominantes. Confirmando el paso desde modelos punitivos, que buscan encauzar la moral del individuo sancionando las conductas que se etiquetan como anormales, hacia formas de control más sofisticadas (FOUCAULT, 2003). Un análisis de estos procesos aparece en el estudio de Molina (2014) en el que se observa cómo las personas que quieren perder peso expresan argumentos relativos a incrementar su capacidad productiva y su deseabilidad social. En esta línea, Guthman (2009) recoge testimonios de estudiantes que vinculan el sobrepeso a una debilidad corporal y moral.

\section{Las definiciones expertas de la obesidad y sus efectos socialmente interactivos}

El trabajo de I. Hacking pone sobre la mesa los efectos sociales de la interacción sostenida de las personas con un tipo de categorías científicas definidas para caracterizar problemáticas de los seres humanos. Dichas entidades se constituyen 
por convenciones y prácticas culturales que sólo cobran sentido dentro un marco de racionalidad específico y no universal. Por lo tanto:

Todos nuestros actos están sujetos a descripciones, y los actos que podemos llevar a cabo dependen, en un sentido puramente formal, de las descripciones de que disponemos. Además, las clasificaciones no existen solo en el espacio vacío del lenguaje, sino en las instituciones, las prácticas, las interacciones materiales con las cosas y con otras personas. [...] (HACKING, 2001, p. 64).

La especificidad de estas categorías que denomina interactivas, radica en que generan efectos en torno a las personas que designan. En el caso de la obesidad, ésta es puesta en práctica y divulgada a través de diagnósticos, campañas de prevención y medios de comunicación. Lo más interesante es lo que toca a la huella de estas sucesivas intervenciones en las categorías, convenciones, saberes y prácticas de los individuos, articuladas en sus diferentes formas de organización social.

Lo que Hacking denomina efecto bucle de las categorías interactivas, se extiende entonces de múltiples maneras impregnando las relaciones interpersonales e institucionales, revelando que las personas se comportan como clases interactivas (HACKING, 2001, p. 65). Las consecuencias nocivas del exceso de peso en la salud son algo indiscutiblemente objetivo, especialmente a largo plazo. Ahora bien, el marco de comprensión que subyace a la declinación de la calidad de vida como objetivo deseable, como riesgo potencial, como manifestación de irresponsabilidad, o como objeto de cálculo de costes sanitarios, implican formas de pensar instituidas en las formas individuales, colectivas e institucionales de actuar, cuyos efectos impredecibles pueden rastrearse en la excesiva preocupación por la imagen corporal o en las adaptaciones interesadas y/o eclécticas de las normas nutricionales expertas que ponen en juego las prácticas sostenidas por los sujetos. Así por ejemplo Martín y Moreno (2005) dan cuenta en su trabajo de los solapamientos y ajustes entre las normas médico-nutricionales y los criterios culturales que rigen la alimentación en las clases populares de Andalucía, partiendo de temas como la convivencia doméstica o el valor afectivo-moral de la "buena maternidad".

Conforme a Hacking las múltiples respuestas generadas por conceptos e intervenciones expertas se explicarían porque las personas, además de interactuar con los discursos expertos interactúan con los juicios y valores de su entorno social (HACKING, 2001, p. 64). Esto explicaría que la obesidad no deba entenderse como una construcción social si lo que se quiere es "dignificar" a las personas con dicho 
diagnóstico, sino más bien como un "producto social contingente" en el que entran en juego identidades individuales y colectivas, en torno al cual se puede tratar de ponderar el peso específico de ciertas determinaciones que pueden aligerar el peso de la excesiva responsabilización.

Para finalizar con este autor, queremos destacar que aunque la inclusión de sus ideas parece conducir a un ámbito filosófico, no debería perderse de vista que es justamente a ese nivel donde es preciso un cambio de orientación. En este sentido, si se quiere dar mayor protagonismo a aquellas "caras" del fenómeno menos tenidas en cuenta, sería conveniente comenzar por pluralizar el entendimiento de lo que es la obesidad en toda su extensión y complejidad. Atender a realidades como los procesos psico-sociales que generan los etiquetamientos clínicos, los itinerarios terapéuticos y sociales de las personas diagnosticadas, o al impacto de las representaciones mass-mediáticas, de manera que pueda percibirse que las verdaderas paradojas de la obesidad (PEETERS, 2018) guardan relación con los resultados conseguidos desde las formas prioritarias de intervención en las que insistimos (WARD et al., 2017).

\section{El sobrepeso y los habitus alimentarios}

En otro sentido las ideas de P. Bourdieu también apuntan a un cambio de concepción. Para empezar porque articula en un mismo marco analítico aspectos claramente medibles, como el papel de ciertos determinantes socio-económicos o la presencia de ciertos elementos fisiológicos; con otros que remiten a procesos claramente subjetivos, como las percepciones, los deseos o las creencias.

Bourdieu propugna un análisis que inscribe las acciones y decisiones de los individuos en espacios concretos de saberes y prácticas (campos sociales) en los que ponen en juego diferentes formas de capital: económico, cultural, social y simbólico (BOURDIEU, 1998). Bajo este punto de vista, las conductas alimentarias estarían marcadas por los capitales que poseen las familias, permitiendo apreciar articulaciones entre aspectos nutricionales y estrategias de distinción social. Para ello designa a los marcos que dan cabida a esos juegos de capitales como habitus, describiéndolos como:

[...] principios generadores de prácticas distintas y distintivas - lo que come el obrero y sobre todo su forma de comerlo, el deporte que practica y su manera de practicarlo, sus opiniones políticas y su manera de expresarlas difieren sistemáticamente de lo que consume o de las actividades correspondientes del empresario industrial—; pero también son esquemas clasificatorios, principios de clasificación, principios de visión y de división, 
aficiones, diferentes. Establecen diferencias entre lo que es bueno y lo que es malo, entre lo que está bien y lo que está mal, entre lo que es distinguido y lo que es vulgar, etc., [...] (BOURDIEU, 1997, p. 20).

El habitus se conceptúa como una herramienta capaz de detectar racionalidades prácticas en las que se entrecruzan el género, la edad o la clase social, a la vez que se expresan las disposiciones más profundas de un individuo socialmente definido. Así, la elección de la comida no solo depende de aspectos descriptivos, como los ideales corporales, o de los efectos fisiológicos de ésta, sino de las preferencias incorporadas que constituyen algo así como "culturas somáticas", que se incorporan mediante una: "relación inteligible y necesaria entre unas prácticas y una situación de las que el propio habitus produce el sentido con arreglo a categorías de percepción y apreciación producidas a su vez por una condición objetivamente perceptible" (BOURDIEU, 1998, p. 99).

De modo sintético podría decirse que el análisis de P. Bourdieu constata la fragmentación de las pautas de consumo de alimentos, en base a normas y condiciones materiales ligadas a segmentos de clase. En torno a dichas pautas se constituyen entonces juegos de distinción, que se adaptan o contraponen a los estilos de vida legítimos definidos desde las instancias médicas: "El arte de beber y de comer es sin duda uno de los pocos terrenos que quedan en los que las clases populares se oponen explícitamente al arte de vivir legítimo" (BOURDIEU, 1998, p. 179). El grueso de la producción epidemiológica reciente viene señalando la necesidad de complejizar las relaciones del status social (género, clase, educación) con el problema de la obesidad (NEWTON; BRAITHWAITE; AKINYEMIJU, 2017), y en esa línea los conceptos bourdieuanos parecen productivos de cara a delimitar segmentos de clase y tipos de gusto alimentario, como también para arrojar luz sobre la transversalidad de ciertos representaciones corporales (delgadez) y como son capitalizados en distintos ámbitos sociales (ROBINOVICH et al., 2018).

Cabe destacar también que las nociones del teórico francés hacen posible visibilizar determinadas violencias simbólicas que envuelven las relaciones de género y que no son percibidas por los propios actores, sencillamente porque sus lógicas se hallan inscritas en el sentido común social como una especie de tradiciones o saberes contrastados (BOURDIEU, 1997, p. 173). Al respecto, diversos trabajos han resaltado cómo la responsabilidad de la alimentación familiar sigue recayendo sobre las mujeres atrapadas de algún modo entre representaciones de la buena maternidad, 
su integración socio-laboral, la necesidad de gestionar bien los recursos familiares y el deseo de alimentar de manera sana a su familia:

Esta buena madre, así, dedica toda su jornada al cuidado familiar: éste es su oficio, para el que ha de desarrollar una serie de cualificaciones específicas al mismo tiempo que tácitas: saber cocinar, educar a los hijos, llevar la casa, hacer la compra... (MARTíN; MORENO, 2005, p. 23).

Además, la cuestión de las violencias simbólicas puede conectarse en otro sentido con las caracterizaciones negativas sobre las personas obesas implícitas en los discursos expertos. Diversos estudios empíricos constatan que las personas obesas experimentan rechazo social, problemas de baja autoestima y altos índices de depresión (MORAES; ALMEIDA; SOUZA, 2013), ligando dicho descrédito social con el efecto de la perspectiva biomédica (GIL-ROMO et al.; 2007, p. 53).

\section{La normalización de la dieta y la emergencia de nuevas subjetividades}

Como los anteriores autores, la potencial aportación de M. Foucault a las discusiones en torno a la obesidad radicaría en que ofrece una alternativa epistemológica enriquecedora en términos de complejidad. La estrategia foucaultiana de historización del presente, apunta hacia las relaciones de poder y formas de saber que atraviesan la conformación misma del fenómeno de la obesidad, abriendo puntos de fuga hacia otras configuraciones posibles del fenómeno.

La tradición médica occidental ha problematizado sostenidamente sobre la "gordura" y sus implicaciones corporales y morales, tratando de ofrecer formas de regulación (tipos, cantidades, elaboraciones, ingesta). Pero la configuración actual del problema presenta singularidades que merece la pena señalar. En ambientes predominantemente propicios para engordar, se ha optado por articular marcos de exigencia para los individuos, de un manejo del peso y la forma corporal basados en el dominio responsable de las necesidades fisiológicas y el aprendizaje de las normas dietéticas.

La regulación de la dieta y del peso es articulada, así, sobre la base de una responsabilización de los individuos sobre sí mismos, de una culpabilización frente a los otros y de la exigencia de una verdadera competencia nutricional. Se trata de un doble proceso, de medicalización y de moralización, [...] (GRACIA, 2009, p. 8).

Históricamente una percepción moralmente negativa ha estado presente en los cuestionamientos en torno al sobrepeso, manifestándose actualmente en lo que M. 
Foucault llamaría "las nuevas pastorales de la salud", que sería el resultado de la adaptación de las relaciones de poder que los Estados modernos heredan de la Iglesia judeo-cristiana y despliegan en los sistemas de salubridad (FOUCAULT, 2006, p. 235).

Así, a partir del siglo XVIII se desarrolló un proceso de patologización de determinadas conductas y sujetos sociales, basado en medidas, diagnósticos y otras formas de objetivación clínicas, asentadas en una legitimidad estatal. “[...] la clínica aparecía, para la experiencia del médico, como un nuevo perfil de lo perceptible y de lo enunciable: nueva distribución de los elementos discretos del espacio corporal [...]" (FOUCAULT, 2001, p. 13). En su articulación de voluntades políticas y conocimientos científicos, los procesos de medicalización vehiculaban marcos de comprensión e intervención social planificada, que tenían por centro el gobierno de la vida (FOUCAULT, 2007). En este marco, los individuos son responsabilizados sobre su peso corporal, mientras procedimientos técnicos y terapéuticos van pasando a formar parte de sus vidas cotidianas, resignificándose dentro de narrativas biográficas donde el peso, la apariencia corporal y la identidad personal serán siempre una tarea inacabada, y las herramientas médicas, nutricionales o psicológicas, recursos que se utilizan dependiendo de contextos diversos (PALACIOS; NAVAS, 2016, p. 93).

De ahí que algunos autores entendieran la declaración de una epidemia de obesidad, como una alarma construida con la intención de aumentar la sujeción de individuos y poblaciones a las instancias médicas (BOERO, 2007, p. 14). No obstante, parece que la "metáfora" de la epidemia ha consolidado el biologicismo implícito en las explicaciones oficiales y en las populares, situando el manejo de la apariencia corporal en el centro de la vida social actual. A la vez que se ha enmascarado el papel de los factores estructurales, económicos y ambientales, para poner el énfasis en los efectos individuales, delineando una política de la obesidad. Permitiendo, si cabe, un mayor control de la vida de las personas desde el ámbito médico (MURRAY, 2008, p. 9). Algo preocupante ya que el asentamiento de ciertas convenciones y estrategias de control del peso, conectan este marco con los trastornos de la conducta alimentaria y los aspectos subjetivos que envuelven a la idealización de la imagen corporal de la delgadez como símbolo de belleza.

En síntesis, observamos que surgen formas de organización social que asientan sus estrategias de control, en mecanismos de objetivación biologicistas, por lo que las reflexiones de Foucault sobre la biopolítica, resultan interesantes para comprender las transformaciones que vienen desarrollándose como efectos del 
marco biomédico de abordaje de la obesidad como problema. Por una parte, por la interiorización entre las poblaciones occidentales de conocimientos y percepciones originados en las formas médicas de intervención, hoy pasados por el tamiz de las consideraciones que tienden a situar los estados subjetivos de los pacientes en el centro de las discusiones. Por otro, porque los avances en genética o neurología han supuesto un reforzamiento de dichos posicionamientos biologicistas velando en su conjunto los aspectos socio-culturales. Así distintos trabajos ponen en juego las nociones del autor francés para analizar las racionalidades políticas que subyacen a los intentos de regular los llamados estilos de vida saludables (COVENEY, 2000; 2008; MAYES, 2016), o la incorporación por parte de los sujetos de las intervenciones expertas, más allá de entendimientos maniqueos de las relaciones poder-resistencia (WARIN, 2011; OURAHMOUNE, 2017).

\section{Una síntesis final}

Como cierre del trabajo, nos gustaría poner de relieve las principales ideas que pretendíamos proponer para la reflexión, y que de algún modo transversalizan los aportes de los autores propuestos. En este caso, trataremos de seńalar de manera concisa dichas ideas, con la intención de posibilitar un marco de reflexión y diálogo.

La agencia de los actores sociales: de forma general, tanto los autores propuestos como mucha de la teoría social actual, propugnan una complejización del entendimiento de la capacidad de agencia detentada por los actores que padecen o participan de determinadas problemáticas. Siendo esta es una cuestión muy a tener en cuenta en la reflexión en torno a la obesidad y el sobrepeso. Tanto las concepciones deterministas de ciertos puntos de vista sociales, como las responsabilizadoras de los puntos de vista biomédicos, simplifican en cierta manera esta cuestión. No parece acertado reducir a los actores individuales y colectivos (familias, grupos sociales) a simples entes sujetos a determinaciones sociales, económicas o políticas que les pre-imponen ciertas formas de abordar su alimentación. Del mismo modo que tampoco parece que la cuestión de los malos hábitos alimentarios y las conductas de riesgo, se pueda explicar sencillamente aduciendo una falta de conocimientos, de recursos o distintos tipos de "debilidades" en la voluntad que hace que las personas decidan mal.

Complejizar las cadenas de causalidad: siguiendo este argumento, parece imprescindible complejizar las cadenas de causalidad con las que elaboramos 
explicaciones sobre el fenómeno del sobrepeso y la obesidad. Según se profundiza en el conocimiento y las intervenciones prácticas en torno al problema en sus distintas dimensiones, resulta más claro que es imprescindible abordar la cuestión de la integración de los diferentes tipos de conocimientos, o dicho de otro modo, es necesario avanzar más rápido en la elaboración de modelos realmente bio-psicosociales para potenciar la generación de modelos de intervención más integrales. Sin duda este es el reto más complejo.

La centralidad de las prácticas: a la vista de los autores que proponemos para la reflexión, seguramente el principal aporte que pueden realizar unas teorías sociales actualizadas es centrar el análisis en las prácticas sociales, es decir, en constelaciones locales, concretas, de formas particulares de hacer y pensar sobre el problema, incluyendo como no las prácticas expertas de intervención. Paradójicamente, esta se presenta como la mejor opción para pluralizar en la práctica una definición de epidemia global, que aún falta por desplegar en cuanto a la elaboración de conocimientos empíricos que muestren qué significan la obesidad y el sobrepeso en distintos ámbitos geográficos, en distintos contextos sociales, en descripciones que incluyan como no, los efectos prácticos, buscados o no de las intervenciones expertas o los marcos económico-políticos.

Los efectos de las concepciones e intervenciones expertas: por último, también resulta esencial que los análisis de la problemática del sobrepeso y la obesidad, comiencen a incluir la ponderación de los efectos explícitos e implícitos de las intervenciones expertas. No solo porque está claro que muchas de estas ideas y prácticas terminan por ser asumidas dentro de procesos y condiciones particulares por las poblaciones. Sino porque a la larga, dichos procesos de asunción de la "cultura científica", terminan por actuar como repositorios a partir de los cuales, se generan modelos de prácticas y concepciones sobre los problemas de salud. ${ }^{1}$

\section{Referencias}

ÁLVAREZ, C. R. La obesidad: es mortal, más se puede prevenir y controlar. Revista de la Facultad de Medicina UNAM, v. 53, n. 3, p. 34-43, 2010.

BENGOA, J. M. Historia de la nutrición en salud pública. Anales venezolanos de la Nutrición, v. 16, n. 2, p. 85-96, 2003.

BerTran, M. Acercamiento antropológico de la alimentación y salud en México. Physis: Revista de Saúde Coletiva, v. 20, p. 387-411, 2010. 
BOERO, N. All the news that's fat to print: The American "obesity epidemic" and the media. Qualitative sociology, v. 30, n. 1, p. 41-60, 2007.

BOURDIEU, P. Razones prácticas Sobre la teoría de la acción. Barcelona: Anagrama, 1997. . La Distinción. Criterio y bases sociales del gusto. Barcelona: Taurus, 1998.

BROWN, P. J. Culture and the evolution of obesity. Human Nature, v. 2, n. 1, p. 31-57, 1991.

BROWNELL, K. D. The environment and obesity. In Fairburn, C.G. \& Brownell, K. D. Eating Disorders and Obesity: A Comprehensive Handbook, p. 433-438. New York: Guilford Press, 2002.

COMTE, A.; REYES, R. C. Ensayo de un sistema de politica positiva. Universidad Nacional Autónoma de México, 1979.

CORELlA, D.; COLTELL, O.; ORDOVAS, J.M. Genética y epigenética de la obesidad. Anales de la Real Academia Nacional de Farmacia, v. 82, p. 129-136, 2016.

COVENEY, J. Food, Morals, and Meanings: The pleasure and anxiety of eating. Routledge: New York, 2000.

. The government of girth. Health Sociology Review, v. 17, n. 2, p. 199-213, 2008.

ELIAS, N. El proceso de la civilización: investigaciones sociogenéticas y psicogenéticas. México: Fondo de Cultura Económica, 2015.

FERNÁNDEZDEROTA, A. Movimientos sociales. Una lectura a partir del postestructuralismo. Athenea Digital, n. 14, p. 63-81, 2008.

FISCHLER, C. El (h) omnivoro, El gusto, la cocina y el cuerpo. Barcelona: Anagrama, 1995.

FLORES, J. A. M.; MARIANO, L. J. Nuevas definiciones de evidencia en la Medicina contemporánea: aportes desde la Antropología. Saude e Sociedade, v. 25, p. 43-56, 2016.

FOUCAULT, M. Historia de la sexualidad. 1-La voluntad de saber. México DF: Siglo XXI, 1991. La vida de los Hombres infames: ensayos sobre desviación y dominación. Editorial Altamira, 1996.

. El nacimiento de la clínica. México DF.: Siglo XXI, 2001

, M. Vigilar y castigar. Nacimiento de la prisión. Argentina: Siglo XXI, 2003

. Seguridad, territorio, población. Curso en el College de France 1977-1978. Buenos Aires:

Fondo de Cultura Económica, 2006.

. Nacimiento de la biopolítica. Curso en el Collège de France 1978-1979. Buenos Aires: Fondo de Cultura Económica, 2007.

FRENCH, S. A.; STORY, M.; JEFFREY, R.W. Environmental influences on eating and physical activity. Annual Review Public Health, n. 22, 309-35, 2001. 
GIL-ROMO, S. E. P.; VEGA-GARCÍA, L. A.; ROMERO-JUÁREZ, G. Prácticas alimentarias de mujeres rurales: ¿una nueva percepción del cuerpo? Salud pública de México, v. 49, p. 52-62, 2007. Disponible en: http://www.redalyc.org/articulo.oa?id=10649108. Acceso en: 16 Dic, 2019. GRACIA, M. Comer bien, comer mal: la medicalización del comportamiento alimentario. Salud pública de México, v. 49, p. 236-242, 2007.

. La emergencia de las sociedades obesogénicas o de la obesidad como problema social. Revista de Nutrição, v. 22, n. 1, p. 5-18. 2009.

. Alimentación y cultura en España: una aproximación desde la antropología social. Physis: Revista de Saúde Coletiva, v. 20, p. 357-386, 2010.

GUTHMAN, J. Teaching the politics of obesity: Insights into neoliberal embodiment and contemporary biopolitics. Antipode, v. 41, n. 5, p. 1110-1133, 2009.

HACKING, I. ¿La construcción social de qué? Barcelona: Paidós, 2001.

LAKE, A.; TOWNSHEND, T. Obesogenic environments: exploring the built and food environments. The Journal of the Royal society for the Promotion of Health, v. 126, n. 6, p. $262-$ 267, 2006.

LANGREO, N. A. El sistema alimentario español desde la perspectiva de los sectores productivos (II). Distribución y consumo, v. 82, p. 5-40, 2005.

LÉVI-STRAUSS, C. Lo crudo y lo cocido. Mitológicas I, México: Fondo de Cultura Económica, 1968.

MANZINI, I. L'alimentation et la médecine dans le monde antique. En: Flandrin JL, Montanari M. Histoire de l'alimentation. París: Fayard, 1996.

MARTÍN, E.; MORENO, J. L. Conflicto sobre lo sano. Un estudio de la alimentación en las clases populares en Andalucía. Sevilla: Consejería de Salud, 2005.

MÁRQUEZ, S.; MENEU, R. La medicalización de la vida y sus protagonistas. Gestión clínica y sanitaria, v. 5, n. 2, 47-53, 2003.

MAUSS, M. Sociología y Antropología. Madrid: Etnos, 1971.

MAYES, C. The Biopolitics of Lifestyle: Foucault, Ethics and Healthy Choices. Oxford: Routledge, 2016.

MENNELL, S. All manners of food. Eating and taste in England and France from de Middle Age to the present. Oxford: Basil Blackwell, 2009.

MOLINA, V. N. Construcción de subjetividad en los llamados obesos: un análisis biopolitico en la cirugía bariátrica. Dissertación (Doctorado). Disponible en: http://bibliotecadigital.univalle. edu.co/handle/10893, 2014. Acceso en: 7 Abr 2020.

MONTANARI, M. La comida como cultura. Gijón: Ediciones Trea, 2006. 
MORAES, A.; ALMEIDA, E.; SOUZA, L. Percepçôes de obesos deprimidos sobre os fatores envolvidos na manutenção da sua obesidade: investigação numa unidade do Programa Saúde da Família no município do Rio de Janeiro. Physis: Revista de Saúde Coletiva, v. 23, p. 553-572, 2013. MURRAY, S. Pathologizing "fatness": Medical authority and popular culture. Sociology of sport journal, v. 25, n. 1, p. 7-21, 2008.

MURRIETA, R. S. S. O dilema do papa-chibé: consumo alimentar, nutrição e práticas de intervenção na Ilha de Ituqui, baixo Amazonas, Pará. Revista de Antropologia, v. 41, n. 1, p. 97-150, 1998.

NEEL, J. Diabetes mellitus: a "thrifty" genotype rendered detrimental by "progress"? American journal of human genetics, v. 14, n. 4, p. 353, 1962.

NEWTON, S.; BRAITHWAITE, D.; AKINYEMIJU, T. Socio-economic status over the life course and obesity: Systematic review and meta-analysis, PLoS One, v. 12, n. 5, 2017.

NUNES, M. From application to implication in medical anthropology: political, historical and narrative interpretations of the world of sickness and health. História, Ciências, SaúdeManguinhos, v. 21, n. 2, p. 403-420, 2014.

ORGANIZACIÓN MUNDIAL DE LA SALUD. Obesity: preventing and managing the global epidemic. WHO Technical Report Series, n. 894. Geneva, Switzerland: WHO, 2000. Disponible en https://www.who.int/nutrition/publications/obesity/WHO_TRS_894/en/. Acceso en: 8 Abr 2020.

. Serie de Informes Técnicos 916. Dieta, nutrición y prevención de enfermedades crónicas. Disponible en https://www.who.int/nutrition/publications/obesity/WHO_TRS_916_spa.pdf. Acceso en: 16 Dic. 2019, 2003.

OURAHMOUNE, N. Embodied transformations and food restrictions: The case of medicalized obesity, Journal of Business Research, v. 75, p. 192-201, 2017.

PALACIOS, J.; NAVAS, J. Experiencias, sentidos e identidad personal: narrativas de mujeres con sobrepeso en tratamiento en el sur de España. Revista San Gregorio, n. 13, p. 90-101, 2016. PEETERS, A. Journals should no longer accept 'obesity paradox' articles, International Journal of Obesity v. 42, p. 584-585, 2018.

ROBINOVICH, J. et al. Embodiment of social roles and thinness as a form of capital: A qualitative approach towards understanding female obesity disparities in Chile. Social Science and Medicine, v. 201. p. 80-86, 2018.

SAGUY, A. C.; RILEY, K. W. Weighing Both Sides. Morality, Mortality, and Framing Contests over Obesity. Journal of Health Politics, Policy and Law, v. 30, n. 5, p. 869-921, 2005.

SATO, P. M. et al. The use of Pierre Bourdieu's distinction concepts in scientific articles studying food and eating: A narrative review. Appetite, v. 96, p. 174-186, 2016 
SWINBURN, B.; EGGER, G.; RAZA, F. Dissecting obesogenic environments: the development and application of a framework for identifying and prioritizing environmental interventions for obesity. Preventive medicine, v. 29, n. 6, p. 563-570, 1999.

ULIJASZEK, S. J.; LOFINK, H. Obesity in biocultural perspective. Annual Review Anthropology v. 35, p. 337-360, 2006.

WARD, D. et al. Strength of obesity prevention interventions in early care and education settings: A systematic review. Preventive Medicine, v. 95, p. 37-52, 2017.

WARIN, M. Foucault's progeny: Jamie Oliver and the art of governing obesity, Social Theory \&. Health, v. 9, p. 24-40, 2011.

\section{Nota}

${ }^{1}$ J. P. H. Alcaraz: revisión bibliográfica, diseño, delimitación de objetivos y redacción del trabajo. J. P. Ramírez: revisión bibliográfica, diseño, delimitación de objetivos y redacción del trabajo. M. E. L. Peinado: revisión bibliográfica, diseño, delimitación de objetivos y redacción del trabajo. 


\section{Abstract}

\section{Updating the sociocultural approaches to obesity: proposals from Hacking, Bourdieu and Foucault}

The increase in obesity rates and their negative consequences, both individually and collectively, call for the need for new scientific approaches, which allow for a multidisciplinary approach that integrates the biological and sociocultural, exploring in depth both aspects objectives as subjective of this problem. In this connection, in this work we propose a set of theoretical reflections, which can serve as a critical framework for updating sociocultural contributions to the scientific debates on obesity and overweight. In order to that first we will review the main characteristics of the biological and sociocultural approaches on the subject. And then we will propose this set of reflections, performing an analysis of the concepts of three critical social theorists, seen as potentially fruitful but little used models in this type of subject by different reasons.

> Keywords: obesity; scientific discussions; sociocultural approaches; Hacking; Bourdieu; Foucault; theoretical refreshing. 


\section{Resumo}

\section{Atualizando abordagens socioculturais da} obesidade: propostas baseadas em Hacking, Bourdieu e Foucault

$\mathrm{O}$ aumento das taxas de obesidade e suas consequências negativas, tanto individuais como coletivas, exigem novos enfoques científicos que permitam uma abordagem multidisciplinar que integre fatores biológicos e socioculturais, explorando em profundidade tanto aspectos objetivos como subjetivos desse problema. Neste sentido, no presente trabalho propomos um conjunto de reflexóes teóricas que pode servir de marco crítico atualizado de contribuiçóes de corte sociocultural para ou debate científico sobre obesidade e sobrepeso. Para isso, primeiro revisaremos as principais características dos enfoques biológicos e socioculturais deste tema. Depois, passaremos a propor este conjunto de reflexôes, realizando uma análise dos conceitos de três teóricos sociais de corte crítico, vistos como modelos potencialmente frutífero que, no entanto, por diferentes motivos, são pouco utilizados nesta temática.

Palavras-chave: obesidade; debates científicos; enfoques socioculturais; Hacking; Bourdieu; Foucault; atualização teórica. 